\title{
BRONCHOALVEOLAR CARCINOMA: CLINICAL, RADIOLOGIC, AND PATHOLOGIC FACTORS AND SURVIVAL
}

Kenichi Okubo, MD

Eugene J. Mark, MD

Douglas Flieder, MD

John C. Wain, MD

Cameron D. Wright, MD

Ashby C. Moncure, MD

Hermes C. Grillo, MD

Douglas J. Mathisen, MD
Background: The principal feature of bronchoalveolar carcinoma is that it spreads along airways or aerogenously with multifocality, but many issues are unresolved. Methods: We studied 119 patients with pathologically confirmed bronchoalveolar carcinoma. Symptoms, smoking status, radiologic findings, the size of tumor, operative procedures, and complications were reviewed. We studied the pathologic features: presence or absence of aerogenous spread, patterns of growth, cell type, nuclear grade, mitosis, rate of bronchoalveolar carcinoma in adenocarcinoma, and lymphocyte infiltration. The correlation among clinical, radiologic, and pathologic findings was examined, and the factors affecting survival were analyzed. Results: Symptomatic patients had more infiltrative radiographic features, and asymptomatic patients tended to have more mass-like features $(P<.0001)$. Tumors with radiographically infiltrating lesions tended to have mucinous histologic features $(P=\mathbf{. 0 0 6})$. Tumors with mass lesions by radiograph tended to have nonmucinous and sclerosing histologic features $(P=.003)$. Aerogenous spread was seen in $94 \%$ of specimens. The presence of a variety of cell types suggested multiple clonal origin. The overall survival in those patients undergoing resection was $69.1 \%$ at 5 years and $56.5 \%$ at 10 years. The significant factors affecting survival were radiologic presence of a mass or infiltrate, pathologic findings of the presence of sclerosis, association with a scar, the rate of bronchoalveolar carcinoma in adenocarcinoma, lymphocyte infiltration grade, nodal involvement, and status of complete resection. Mitosis or nuclear grade of tumor cells did not correlate with survival. Conclusions: Bronchoalveolar carcinoma showed good overall survival with appropriate surgical procedures. Certain radiologic or pathologic findings correlated with survival. These findings may enhance the ability to predict long-term survival. (J Thorac Cardiovasc Surg 1999;118: 702-9)
$\mathrm{B}$ ronchoalveolar carcinoma (BAC) is considered a subtype of adenocarcinoma. ${ }^{1}$ Since first described in $1876,{ }^{2}$ it has been called by a variety of different names (alveolar cell carcinoma, bronchiolar carcinoma, and BAC). The characteristic pathologic features of $\mathrm{BAC}$ are the presence of aerogenous spread and evidence of advancement along the alveolar wall. BAC can coexist with adenocarcinoma, and overlap within the same tumor often occurs. Prognosis for BAC was origi-

From the Thoracic Surgery and Pathology Departments, Massachusetts General Hospital, Boston, Mass.

Read at the Seventy-eighth Annual Meeting of The American Association for Thoracic Surgery, Boston, Mass, May 3-6, 1998.

Received for publication May 8, 1998; revisions requested July 6, 1998; revisions received May 7, 1999; accepted for publication May 7, 1999. nally thought to be poor ${ }^{3}$; however, more recent reports demonstrate a good prognosis for localized neoplasms. ${ }^{4-6}$

There have been claims of correlation between certain clinical, radiographic, and pathologic findings and survival. $^{6-8}$ Some researchers have claimed a predilection for BAC in women and no correlation to cigarette smoking. Because of these claims with conflicting reports, we reviewed our experience with BAC to see whether we could corroborate or refute some of these claims.

\footnotetext{
Address for reprints: Douglas J. Mathisen, MD, Thoracic Surgery, Blake 1570, Massachusetts General Hospital, Fruit St, Boston, MA 02114 .

Copyright $($ C) 1999 by Mosby, Inc.
}

$0022-5223 / 99 \$ 8.00+0 \quad \mathbf{1 2 / 6 / 9 9 9 3 5}$ 
Table I. Operative procedures

\begin{tabular}{lr}
\hline Procedure & $N$ \\
\hline First operation & 114 \\
Lobectomy & 73 \\
+Wedge resection & 5 \\
+Segmentectomy & 2 \\
+Chest wall & 1 \\
+Diaphragm & 1 \\
Bilobectomy & 6 \\
Pneumonectomy & 7 \\
+Diaphragm & 1 \\
+Sleeve pneumonectomy & 1 \\
Segmentectomy & 8 \\
+Wedge resection & 1 \\
Wedge resection & 13 \\
Open lung biopsy & 7 \\
Subsequent operations & 11 \\
Completion pneumonectomy & 3 \\
Lobectomy & 4 \\
+Wedge & 1 \\
Segmentectomy & 2 \\
Wedge resection & 1 \\
\hline
\end{tabular}

\section{Methods}

We studied 119 patients with BAC histologically confirmed at the Massachusetts General Hospital from 1977 to 1995. BAC was defined, according to World Health Organization criteria, as a variant of adenocarcinoma in which more than one half of the malignant cells grew along the alveolar walls and within alveolar spaces with preservation of the alveolar framework of the lung. The records of all patients were reviewed for clinical characteristics: symptoms, smoking status, method of diagnosis, operative findings and procedure, complications, and long-term survival. All living patients or family were contacted by telephone. A lung resection was considered curative when all known BAC was removed and no microscopic tumor was left in the surgical margin. Operative death was defined as all deaths occurring within 30 days of operation.

All chest radiologic findings were reviewed to determine the characteristics because computed tomography scans were not available for all patients during the period of study. Radiographic lesions were classified according to the presence of a mass or infiltrate. The lesion was considered an infiltrate when it was a poorly delineated opacity without defined borders. The infiltrative lesions were subclassified according to unilateral or bilateral, and within one or more lobes. The mass lesions were subclassified as single or multiple, and larger or smaller than $3 \mathrm{~cm}$, if single.

All solitary neoplasms were postoperatively staged according to the current International TNM Classification System for Lung Cancer. ${ }^{9}$ When multiple lesions were resected, the patients were separated simply as unilateral or bilateral. Gross and microscopic pathologic features were studied. Grossly, the tumors were characterized according to the following features: solitary, diffuse, or multifocal; well-defined
Table II. Pathologic findings $(n=119)$

\begin{tabular}{lc}
\hline Findings & $N$ \\
\hline Histologic evidence & \\
Mucinous & 26 \\
Nonmucinous & 82 \\
Mucinous + nonmucinous & 11 \\
Sclerosing & 96 \\
Associated scar & 72 \\
Cell type & \\
Clara & 69 \\
Endocervical mucinous & 9 \\
Type II & 6 \\
Colonic mucinous & 1 \\
Mixed & 34 \\
BAC \% & \\
$50 \%$ & 20 \\
$60 \%$ & 23 \\
$70 \%$ & 14 \\
$80 \%$ & 22 \\
$90 \%$ & 23 \\
$100 \%$ & 17 \\
Nuclear grade & 38 \\
Well-differentiated & 41 \\
Moderate & 22 \\
Poor & \\
Mitoses (per 10 high-power fields) & \\
0 & 21 \\
1 & \\
2 & \\
Lymphocyte infiltration & \\
Grade 0 & \\
Grade 1 & \\
Grade 2 & \\
Grade 3 & \\
\hline
\end{tabular}

nodule, ill-defined single mass, or pneumonic. Microscopically, 8 features were evaluated: tumor border (sharp, ill defined, mixed); aerogenous spread (yes or no); subtype of adenocarcinoma (acinar, papillary, bronchoalveolar, and the percent calculated accordingly); pattern (mucinous, mucinous-sclerosing, nonmucinous, nonmucinous-sclerosing); cell type (type II pneumocyte, Clara cell, colonic mucinous, endocervical mucinous, or mixed); nuclear grade (well differentiated, moderately differentiated, poorly differentiated); mitotic activity (number per 10 high-power fields); and the degree of lymphocytic inflammation (quantitating from 0 through 3).

Clinical features of sex, symptoms, and smoking status were analyzed for the incidence of disease. The relationship between clinical, radiologic, and pathologic findings were examined. Symptoms, smoking status, the presence of a mass or infiltrate, mucinous or nonmucinous, sclerosing or nonsclerosing, and the percent of BAC cells were chosen and examined for the relation to each other. Factors were then analyzed according to their impact on survival.

Statistics. The survival analysis was estimated by the Kaplan-Meier actuarial method, ${ }^{10}$ with the date of initial 
Table III. Stage of tumor in patients undergoing

\begin{tabular}{lc}
\multicolumn{2}{l}{ resection } \\
\hline Stage & $N$ \\
\hline I & \\
T1 N0 & 55 \\
T2 N0 & 26 \\
II & \\
T1 N1 & 3 \\
T2 N1 & 4 \\
III & \\
T3 N0 & 2 \\
T1 N2 & 2 \\
IV & \\
T1 N0 M1 & 1 \\
Multiple & 14 \\
\hline
\end{tabular}

operation as the starting date. The difference of variables on survival was analyzed with the log-rank rest for cosorted data. ${ }^{11}$ Qualitative data were analyzed with $\chi^{2}$ tests. Cox multivariate analysis was used to evaluate all factors. All reported $P$ values are 2-tailed.

\section{Results}

Clinical findings. Of 119 patients available for study, 44 were men and 75 were women. Ages ranged from 15 to 83 years (mean, $65.5 \pm 11.7$ years) in men and from 29 to 75 years (mean, $63.1 \pm 9.7$ years) in women. Eighty-five patients $(71 \%)$ were asymptomatic. Cough, dyspnea, and sputum production were the most common symptoms. Eighty patients $(68 \%)$ were current or former smokers, and 39 patients were nonsmokers.

Radiologic findings. Chest radiographs showed a mass lesion in 94 patients and infiltrating lesion in 25 patients. Among mass lesions, a single lesion was present in 80 patients $(\leq 3 \mathrm{~cm}$ in 58 patients and $>3 \mathrm{~cm}$ in 22 patients). Multiple lesions were present in 14 patients (unilateral in 10 patients and bilateral in 4 patients). Among infiltrating lesions, 15 patients had unilateral lesions and 10 patients had bilateral lesions.

Method of diagnosis. The diagnosis of malignancy was made by sputum cytologic evaluation in 4 patients, bronchoscopic examination in 12 patients, needle aspiration biopsy in 40 patients, prior brain surgical procedure in 1 patient, and surgical resection in 62 patients.

Operative procedure. One hundred seven patients underwent surgical resection; 7 patients had open lung biopsies, and 5 patients had transbronchial biopsy only. Five patients underwent multiple surgical procedures for tumor recurrence. The operative procedure for the first, second, or subsequent resections are shown in Table I. The most common procedure was lobectomy (64\%, first surgical procedure).
Table IV. Clinical, radiographic, and pathologic correlations

\begin{tabular}{lccc}
\hline & Mass (\%) & Infiltrate (\%) & P value \\
\hline Symptoms & 16 & 76 & $<.0001$ \\
Asymptomatic & 84 & 24 & \\
Mucinous & 16 & 44 & .0060 \\
Mixed & 9 & 12 & \\
Nonmucinous & 75 & 44 & .0032 \\
Sclerosing & 86 & 60 & \\
Nonsclerosing & 14 & 40 & \\
\hline
\end{tabular}

Morbidity and mortality. There were 3 operative deaths (mortality, 2.4\%). Cause of death was cerebral vascular accident at day 7 , sudden cardiac arrest probably the result of pulmonary embolism or myocardial infarction just after discharge at day 22, and pneumonia followed by sepsis and gastrointestinal bleeding at day 9. Postoperative complications occurred in 34 patients $(27.2 \%)$. One quarter of the complications were major.

Pathologic findings. One hundred two first-time surgical procedures were considered curative. Five patients did not undergo curative resections because of tumor at the resection margins on histologic examination. There were 11 reoperations in 5 patients; 8 were deemed curative. Of the 5 patients undergoing multiple operations, 3 patients had similar pathologic findings as the first operation, and 2 patients had different findings. The pathologic findings are listed in Table II. Findings of aerogenous spreading were seen in 113 of 119 patients. Most of the tumors in this series did not show signs of mucin production (82/119 tumors). Most of the tumors had evidence of sclerosis present or were associated with the presence of a scar. The most common cell type seen was the Clara cell. Other cells identified included endocervical mucinous, type II pneumocyte, or colonic mucinous cells. Approximately $29 \%$ of tumors had a mixture of different types of cells present. A pure bronchoalveolar cell pattern was seen in only 17 of 119 patients. Most tumors were a mixture of bronchoalveolar cell carcinoma and adenocarcinoma with percentages of BAC ranging from $50 \%$ to $90 \%$. Most tumors were graded as moderately differentiated (83/119 tumors) with comparable numbers of well and poorly differentiated tumors. Sixty-eight patients had no mitotic activity seen on 10 high-power microscopic examinations; 41 patients had only 1 mitosis seen per 10 high-power fields, and 7 patients had 2 or more mitoses per 10 high-power fields. A qualitative assessment was made of the presence of lymphocyte infiltration into the tumors. Eighty-five patients had grade 0 to 


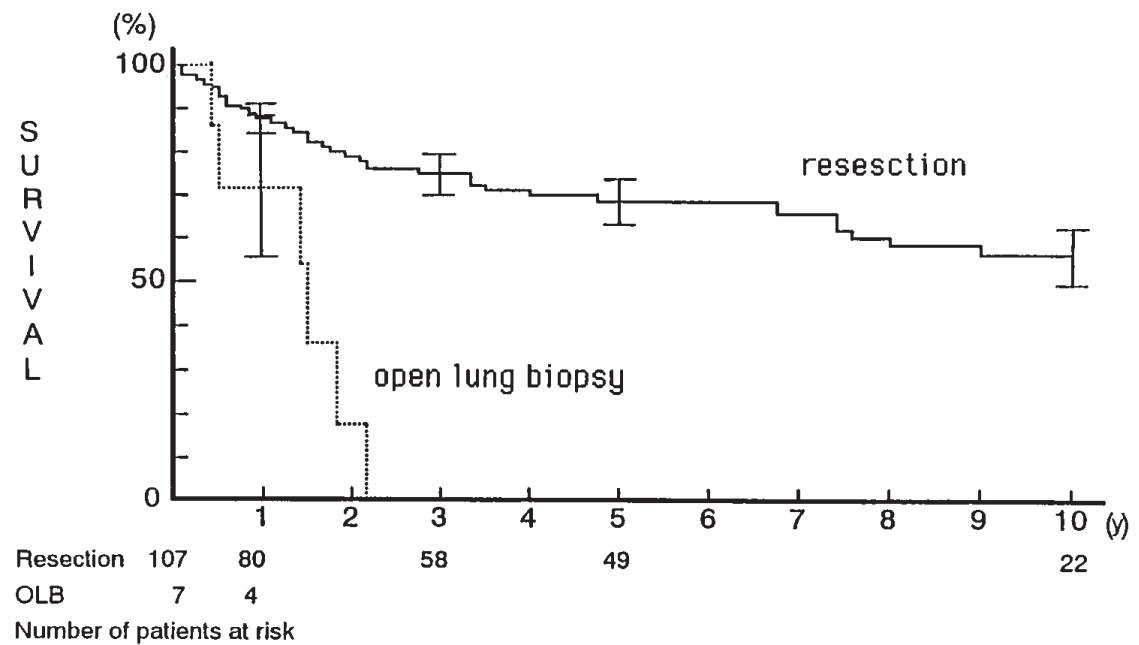

Fig 1. Overall survival for patients undergoing surgical resection $(\mathrm{n}=107$ patients $)$ or open lung biopsy $(O L B ; \mathrm{n}=$ 7 patients).

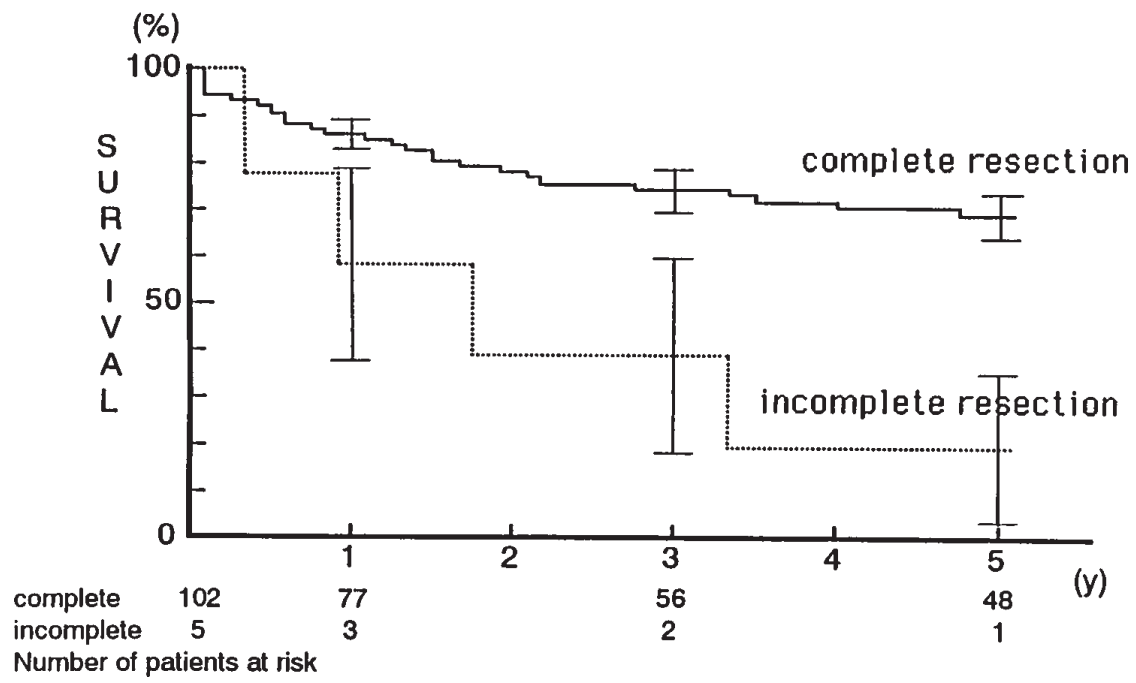

Fig 2. Survival for complete ( $\mathrm{n}=102$ resections) versus incomplete resection ( $\mathrm{n}=5$ resections). Complete resection showed better prognosis than incomplete resection $(P<.01)$

2 lymphocyte infiltration, and 22 of 119 patients had grade 3 lymphocyte infiltration.

Pathologic staging of tumor. Pathologic staging was performed for each patient undergoing surgical resection (Table III). Eight-two percent of patients were either surgical stage I or II.

Incidence of clinical factors. There was no difference in the average age between men and women. Thirty-six of the men $(82 \%)$ were smokers, whereas 44 of the women $(59 \%)$ were smokers $(P=.016)$. There was no significant relation between the presence of symptoms and smoking status.
Correlations among clinical, radiographic, and pathologic findings. Symptomatic patients $(76 \%)$ more often had infiltrative radiographic features, and asymptomatic patients more often had mass lesions (84\%; $P<.0001)$. There was no correlation between smoking status and radiographic findings (Table IV). Infiltrative radiologic findings correlated with mucinous histologic findings $(P=.0060)$, and mass lesions correlated with nonmucinous histologic findings and the presence of sclerosis $(P=.0032)$. The pathologic finding of $100 \%$ BAC correlated with nonsmokers and infiltrative lesions. No other significant correlations 


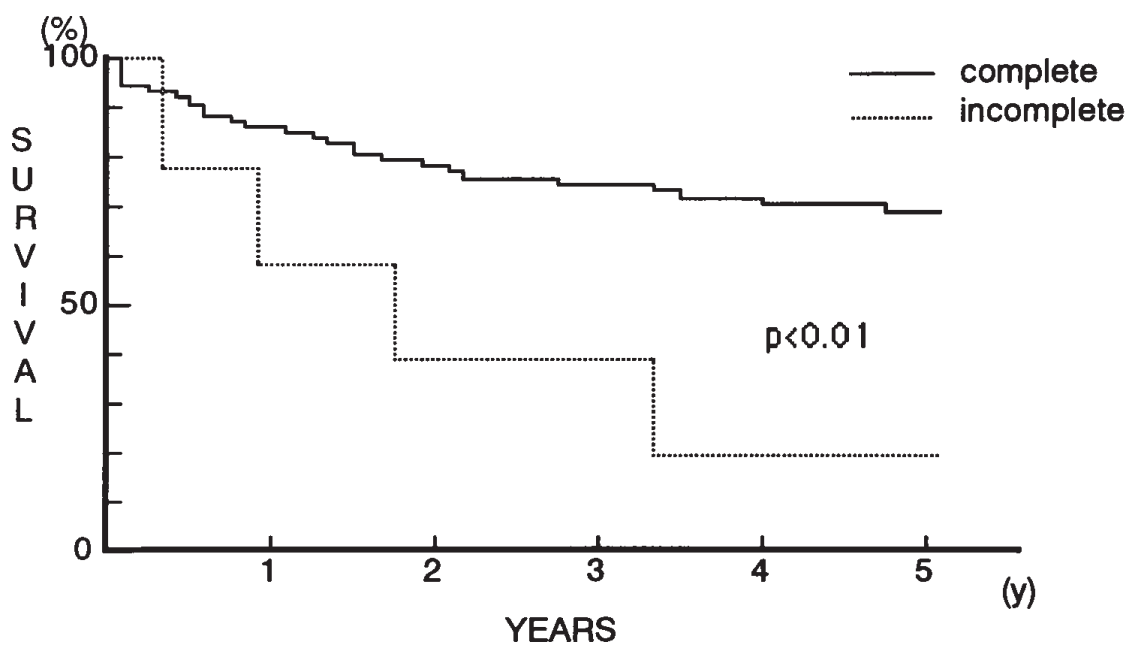

Fig 3. Survival by operative procedure. Segmentectomy $(n=8)$ showed better prognosis than wedge resection $(n$ $=13 ; P<.01)$.

Table V. Factors adversely affecting survival $(n=107)$

\begin{tabular}{ll}
\hline Factors & P value \\
\hline Clinical features & \\
Symptomatic versus asymptomatic & .05 \\
Smoking versus nonsmoking & .2 \\
Radiologic findings & \\
Infiltrate versus mass & .004 \\
Single versus multiple mass & .19 \\
$\leq 3$ cm versus > cm (single mass) & .2 \\
Pathologic findings & .8 \\
Aerogenous spread & .7 \\
Mucinous versus nonmucinous & .001 \\
Nonsclerosing versus sclerosing & .02 \\
No scar versus scar & .4 \\
Mitosis 0 and 1 vs 2 & .6 \\
Nuclear grade (well/moderate/poor) & .02 \\
Rate of BAC 100\% versus 50\%-90\% & .03 \\
Lymphocyte grade 0+1 + 2 versus 3 & .001 \\
N1+2 versus N0 & .2 \\
T1 N0 versus T2 N0 (N0) & $<.001$ \\
Margin positive versus negative & \\
\hline
\end{tabular}

existed among the other clinical, radiologic, or pathologic factors.

Factors affecting survival. Median duration of follow-up among the surviving patients after operation was 6.9 years. Overall survival of patients who underwent any surgical procedure was $64.8 \%$ at 5 years and $53.0 \%$ at 10 years. The survival of the patients undergoing surgical resection was $69.1 \%$ at 5 years and $56.5 \%$ at 10 years (Fig 1). No patient undergoing open lung biopsy survived more than $2 \frac{1}{2}$ years. All the patients undergoing multiple operations survived more than 50 months after the initial operation. The existence of malignant cells in the resected margin correlated with a poor prognosis $(P<.01$; Fig 2$)$. The survival according to operative procedure is seen in Fig 3. Segmentectomy showed a statistically significant better prognosis than wedge resection $(P=.022)$. The factors affecting survival by univariate analysis (Table $\mathrm{V}$ ) were radiographic findings of mass or infiltrate (Fig 4), nodal involvement, pathologic findings of sclerosis, and association with scar. The percent of BAC (Fig 5) showed a significant difference in survival; $100 \%$ presence of BAC showed a worse prognosis than tumors with a mixture of BAC and adenocarcinoma $(P<.05)$. The grade of lymphocyte infiltration (Fig 6) showed significant differences in survival. Patients with grade 3 lymphocyte infiltration had a better prognosis than those with grade 0,1 , or $2(P<.01)$. Multivariate analysis showed that nodal involvement and the radiologic finding of an infiltrative process had a negative impact on survival and that the presence of a mass lesion had a positive impact on survival.

\section{Discussion}

BAC represents between $1 \%$ and $9 \%$ of lung cancers in most series. ${ }^{4,12,13,15}$ Confusion has surrounded many aspects of this tumor. Much of the confusion relates to the relative infrequency of this tumor, disagreement among pathologists about the pathologic findings, and lack of correlation between clinical, radiographic, and pathologic findings and their impact on survival.

In our series, we found a nearly 2:1 ratio of women 


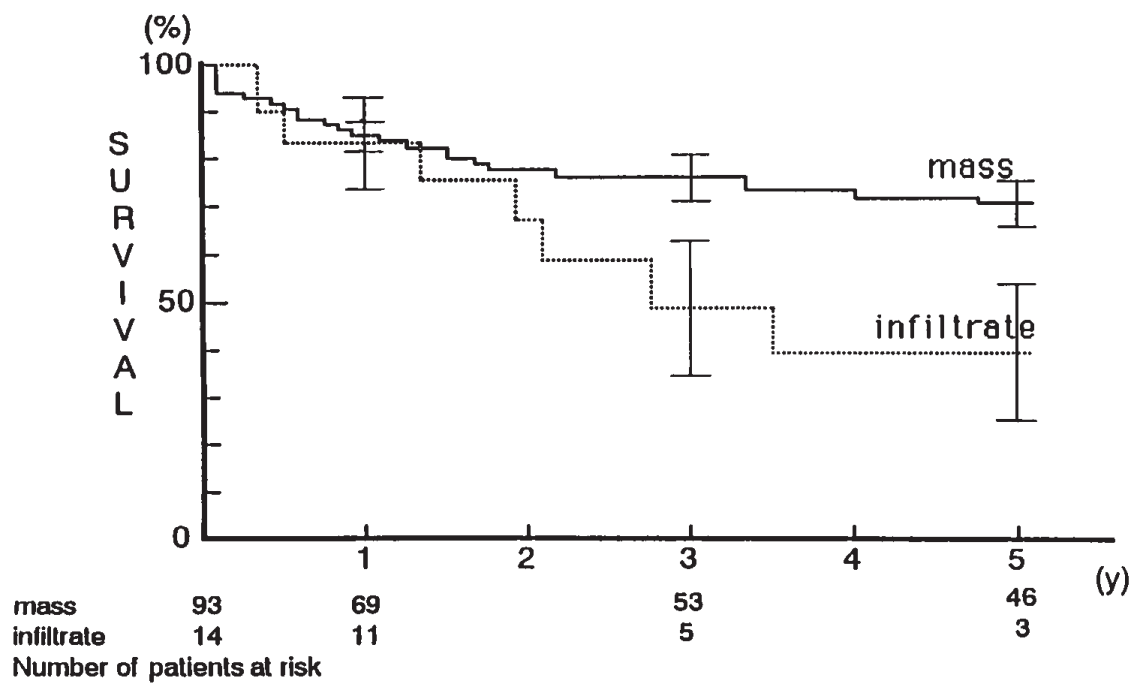

Fig 4. Survival according to the presence of a mass or infiltrate on radiograph. Radiologic mass $(n=93)$ showed better prognosis than infiltrate $(\mathrm{n}=14 ; P<.004)$.

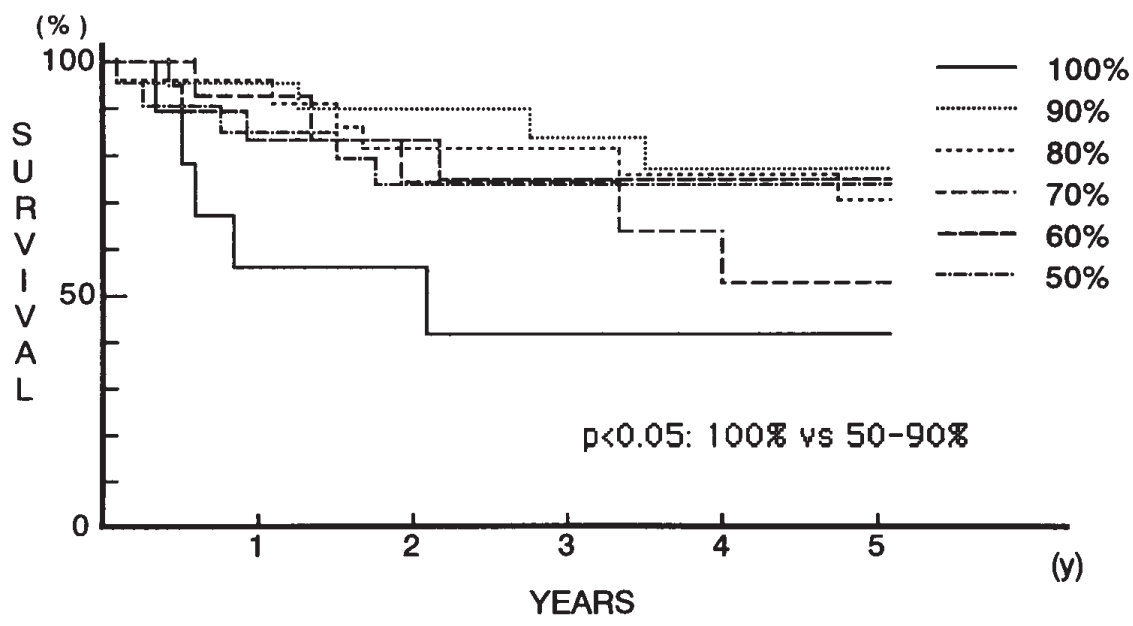

Fig 5. Survival according to percent BAC seen in tumor: $100 \%$ BAC showed worse prognosis than $50 \%$ to $90 \%$ BAC $(P<.05)$; no significant differences were seen among $50 \%$ to $90 \%$ BAC.

to men. This has been reported by others but not all authors. ${ }^{42,13,15}$ Because most of our patients had solitary pulmonary nodules, nearly $72 \%$ were asymptomatic. Of these patients having symptoms, $56 \%$ had evidence of infiltrative lesions on radiographs. Sixtyeight percent of the patients in our series were smokers, but this did not have an impact on survival.

The most common operation in our series was lobectomy. Some of the neoplasms were more advanced and required more extensive procedures. Small peripheral lesions were managed by lobectomy, segmentectomy, or wedge resection. There was a statistically significant difference in survival between segmentectomy and wedge resection. Because of the patterns of spread of BAC along alveolar walls and by aerogenous routes, it should be recommended that lobectomy or segmentectomy is preferable for small peripheral neoplasms.

The most common cell type seen was the Clara cell. Other cell types or mixture of cells were identified. This suggests a multiclonal origin of this tumor. The pattern of multiple cells of origin argues that multiple separate independent primary tumors occur in contiguous foci. This may explain the differences in histologic evidence seen in some of the recurrent tumors and the 


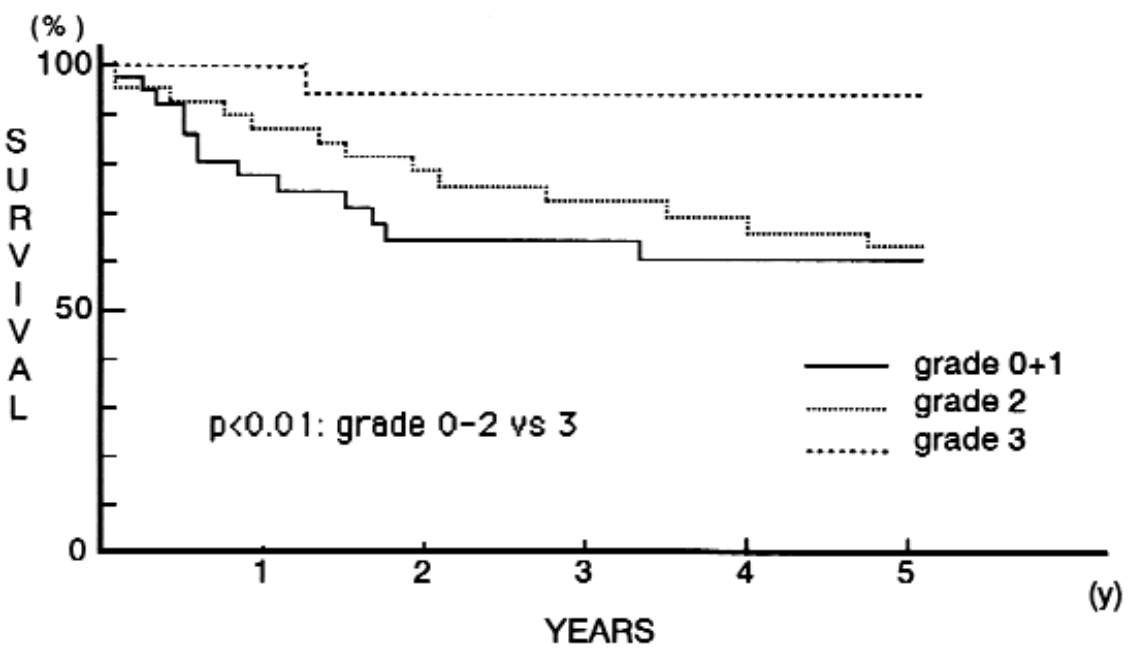

Fig 6. Survival according to grade of lymphocyte infiltration into the tumor. Patients with grade 3 lymphocyte showed better prognosis than those patients with grade 0 through $2(P<.01)$. No significant difference was seen between grade $0+1$ and grade 2 .

frequently observed admixture with typical findings of adenocarcinoma. ${ }^{14-16} \mathrm{~A}$ pure BAC pattern was seen in only 17 of 119 patients, correlating with an infiltrative pattern on radiograph. This small subset of patients correlated with a worse prognosis than those patients with other percentages of BAC. Most patients in this series showed histologic signs of sclerosis in the presence of a scar, and most were nonmucinous as well. This has been reported by others. ${ }^{6}$ The presence of sclerosis and a scar had a positive correlation with survival, but no impact on survival was found among those tumors that showed signs of mucin production contrary to what others have reported. ${ }^{6,17}$ The differences in pulmonic involvement in neoplasms with mucinous, nonmucinous, and sclerosis present histologically are reported to be due to differences in basement membrane integrity and type IV collagenase activity, which destroys the basement membrane and facilitates the spread of tumor through the lymphatic duct. ${ }^{18}$ The other interesting histologic finding was the positive correlation between grade 3 lymphocyte infiltration and survival. Whether this finding implies a beneficial host immunologic reaction to the tumor is purely speculation. Further evaluation of this finding is warranted.

The overwhelming majority of our patients had stage I or II neoplasms that represented solitary nodules. This accounts for the 5- and 10-year survival figures of $69 \%$ and $56 \%$, respectively, in patients undergoing surgical resection. It was important to achieve complete surgical resection. The 5-year survival for completely resected patients was $71 \%$ and only $20 \%$ for incom- plete resections ( $1 / 5$ patients; $P<.01)$. Positive hilar or mediastinal lymph nodes occurred in only $10 \%$ of our patients but was a statistically significant factor adversely affecting survival.

Overall, we were able to develop a favorable profile for patients with BAC. Patients that had no symptoms and a mass lesion on chest radiograph have a better prognosis. Conversely, those patients with an infiltrative picture and symptoms had a worse prognosis. Histologically, the presence of sclerosis and a scar, abundant infiltration with lymphocytes, and no evidence of lymph node metastases are associated with a more favorable prognosis. Patients undergoing complete surgical resection with these findings have a good prognosis.

\section{REFERENCES}

1. World Health Organization. Histological typing of lung tumors. In: International histological classification of tumors. 2nd ed. Geneva: World Health Organization, 1981.

2. Malassez L. Examen histologic d'un cas de cancer encephaloide de poumon (epithelioma). Arch Physiol Norm Pathol 1876;3:353-72.

3. Delarue NC, Graham EA. Alveolar cell carcinoma of the lung (pulmonary adenomatosis Jagziekte?). J Thorac Cardiovasc Surg 1949;18:237-51.

4. Greco RJ, Steiner RM, Goldman S, Cotler H, Patchfsky A, Cohn HE. Bronchoalveolar cell carcinoma of the lung. Ann Thorac Surg 1986;41:652-6.

5. Harpole DH, Bigelow C, Young WG, Wolfe WG, Sabiston DC. Alveolar cell carcinoma of the lung: a retrospective analysis of 205 patients. Ann Thorac Surg 1988;46:502-7.

6. Daly RC, Trastek VF, Pairolero PC, Murtaugh PA, Huang MS, Allen MS, et al. Bronchoalveolar carcinoma: factors affecting survival. Ann Thorac Surg 1991;51:368-77. 
7. Barsky SH, Cameron R, Osann KE, Tomita D, Holmes EC. Rising incidence of bronchioloalveolar lung carcinoma and its unique clinicopathologic features. Cancer 1994;73:1163-70.

8. Hsu C, Chen C, Hsu N. Bronchioloalveolar carcinoma. J Thorac Cardiovasc Surg 1995;110:374-81.

9. Mountain CF. A new international staging system for lung cancer. Chest 1986;89:225S-33S.

10. Kaplan EL, Meier P. Nonparametric estimation from incomplete observations. J Am Stat Assoc 1958;53:457-81.

11. Cox DR. Regression models and life-tables. J R Stat Soc [B] 1972;34:187-220.

12. Rossing TH, Rossing RG. Survival in lung cancer: an analysis of the effects of age, sex, resectability, and histiopathologic type. Am Rev Respir Dis 1982;126:771-7.

13. Heikkila I. Results of surgical treatment in bronchioloalveolar carcinoma. Ann Chir Gynaecol 1986;75:183-91.

14. Liebow A. Bronchiolar-alveolar carcinoma. Adv Intern Med 1960; 10:329-58.

15. Edgerton F, Rao U, Takita H, Vinccent RG. Bronchioalveolar carcinoma: a clinical overview and bibliography. Oncology 1981; 38:269-73.

16. Clayton F. Bronchioloalveolar carcinomas: cell types, patterns of growth and prognostic correlates. Cancer 1986;57:1555-64.

17. Mainning JT, Spjut JH, Tschen JA. Bronchioalveolar carcinoma: the significance of two histologic types. Cancer 1984;54:525-34.

18. Ohori NP, Yousem SA, Griggin J, Stanis K. Comparison of extracellular matrix antigens in subtypes of bronchioloalveolar carcinoma and conventional pulmonary adenocarcinoma: an immunohistochemical study. Am J Surg Pathol 1992;16:675-86.

\section{Discussion}

Dr Mark S. Allen (Rochester, Minn). Since the initial description of BAC of the lung in 1876, there has been a considerable amount of controversy about this disease. It does not seem to behave like a typical bronchogenic carcinoma. The biology differs from a typical lung cancer because it seems to be slow growing and may have a multicentric origin and does not commonly metastasize to the lymphatics. However, there exists a subset of patients in whom this disease, unfortunately, behaves quite similarly to a typical bronchogenic carcinoma, with a rapid demise despite our best management. I hope that, as the genetic defects are found that transform cells into their malignant behavior, we will begin to understand the differences in these various cancers.

Until this technologic breakthrough occurs, we will have to rely on retrospective reviews such as this to learn about this disease. This article has many similarities to a paper presented at the meeting of the Southern Thoracic Surgical Association in 1990 by Dr Richard Daly from our own institution.

The demographics of the patients with BAC are very similar in the 2 reports; most had a single nodule and were asymptomatic. The operation most commonly performed is a lobectomy, and the mortality rate is quite low. A high percentage of the patients have stage I disease, and their survival is quite good compared with the average patient with bronchogenic carcinoma.

I have 3 questions. In our series we had quite a few patients who had recurrence after 5 years. What is your recommendation for follow-up of this group of patients? Do you obtain a periodic computed axial tomographic scan or do you obtain periodic chest x-ray films only? Do you use sputum cytologic findings in any way to observe these patients?

My second question concerns patients who have a mass on one side that is diagnosed as BAC but have infiltrative lesions on the other side. Do you recommend resecting the larger lesion first and closely monitoring the contralateral lesion, or do you try and diagnose the smaller contralateral lesions first?

Finally, do you think that this is a multicentric disease or do you think that, in patients who have multiple sites, they all originated from one focus?

Dr Okubo. With respect to the third question, I think the tumor is multicentric because of the variety of cell types, including Clara cell, type II pneumocyte. This neoplasm shows a variety of mixed cell types, so I think it is multicentric.

With respect to the second question, we individualize the management of bilateral lesions. If the presence of contralateral disease would contraindicate surgical procedures, we would perform a biopsy of it first. For other reasons we might monitor them or resect at a later date.

Usually postoperative follow-up is done with chest x-ray films and computed axial tomography scans. So, this should be done for follow-up. We have not found sputum cytologic evidence to be useful.

Dr Nasser K. Altorki (New York, NY). I am always troubled by the patient who has a diffuse lobar infiltrate and a diagnosis of BAC. Can you tell me, now knowing what you know about this series, what your strategy has been or will be in the treatment of those patients? Is this a surgical disease?

Dr Okubo. As long as this neoplasm spreads unilaterally, this is a surgical disease. The diffuse infiltrative lesions tend to have a worse prognosis, but they tend to respond poorly to radiation or chemotherapy. There are some patients who have bronchiolar and diffuse disease who should undergo a resection to control symptoms.

Dr Lawrence R. Kaiser (Philadelphia, Pa). Just to follow up on that, how many of the patients in this series who had lobar infiltrates underwent resection, and were any of those patients long-term survivors? The issue of the multicentricity of these lesions is an interesting one. In talking with Rob Garver, who is one of the pulmonologists at the University of Alabama, I believe they have performed transplantations in 7 patients with BAC. Two of those patients have experienced the development of BAC in the transplanted lungs, and they are currently looking to see whether that is of donor or recipient origin.

Are there any long-term survivors with resected lobar infiltrates?

Dr Okubo. Fourteen of 25 infiltrate regions on radiography underwent resections, with a 5 -year survival of $30 \%$ to $40 \%$; but these were not the patients with lobar or an entire lung involved. The diffuse, extensive infiltrative lesions are associated with a much worse prognosis. 